\title{
Highly Adaptive Indian High Security Vehicle Number Plate Recognition
}

\author{
Neha Arora \\ M-Tech Scholar \\ NRI Institute of Information \\ Science and Technology, Bhopal, \\ M.P.
}

\author{
Lalit Jain \\ Research Guide \\ NRI Institute of Information \\ Science and Technology, Bhopal, \\ M.P.
}

\author{
Puran Gour \\ HOD, Department. of Electronics \\ and Communication Engineering \\ NRI Institute of Information \\ Science and Technology, Bhopal, \\ M.P.
}

\begin{abstract}
The recognition techniques are very useful to build the automatic systems, especially it make electronic systems alive as an artificial intelligence devices. The recognition adds functionality of the existing working devices, which enhances the precision as well as reduces manpower, human errors and increases the speed of work. The recognition has many branches like face recognition, object recognition, colour recognition, character recognition etc. In this paper an algorithm is developed to recognize the Indian Vehicle High Security Number Plate with optimum accuracy. The whole recognition procedure has feature database preparation as well as recognition process. The accuracy of the proposed system model is quite high as compared to existing methodologies. The system is very simple and fast to work.
\end{abstract}

\section{Keywords}

High Security Vehicle Number Plate, Character Recognition, Artificial Intelligence and Segmentation.

\section{INTRODUCTION}

A license plate (LP) is the unique identification of a vehicle. License plate is rectangular in shape and size, it contains numbers and alphabets and it must be fixed onto the car body (at least at its backside) and it means a legal license to participate in the public traffic.

In recent years, the number of vehicles has largely increased because of the growth of the urban population and the power of purchasing becomes stronger. Indeed, the manual methods for dealing with a large number of vehicles are consequently difficult. As a result this leads to difficulties in the management of vehicles and also leads to many shortcomings such as the poor working environment, rigorous labor, tedious work, and low effectiveness.

Due to the above mentioned problems and to improve the efficiency of vehicle management and traffic control there is a demand for unmanned license plate recognition system

(LPR) for the automatic identification of vehicles. License number plate recognition is an image processing technology used to identify vehicles by their license number plates only. LPR is one of the important topics of using computer vision and pattern recognition. LPR system has been one of the crucial components of the Intelligent Transportation
Systems (ITS). They have a wide impact in people's life as their scope is to improve transportation safety, mobility and to enhance productivity through the use of advanced technologies. ITS are made up of 16 various types of technology based on systems. These systems can be divided into intelligent infrastructure systems and intelligent vehicle systems. Some of the ITS applications are: Advances in navigation systems, Electronic toll collection systems, provide assistance for safe driving, manage Optimization of traffic management, and Support for emergency vehicle operation. LPR system shell operate fast and accurate enough to satisfy the needs of ITS.

LPR system may be known with the following names:

- Automatic Vehicle Identification (AVI).

- Car Plate Recognition (CPR).

- $\quad$ Automatic Number Plate Recognition (ANPR).

- Vehicle License Plate Recognition (VLPR).

\section{PROPOSED RECOGNITION MODEL}

The Indian high security number plate recognition is the technique which various systems can be automated to work faster on vehicle surveillance and toll management system. In this section a proposed approach is given to recognize high security number plate without any manpower and faster to automate the various vehicle management systems.

The proposed system is having two modules one is training process and second one is recognition process. The training process is shown in the Fig. 2.1 which has input of character template collection. The system will first read the templates and check all the template is in proper dimension or not. Then features of checked templates will be extracted in four different manner i.e. by shifting in upward, shifting in downward, left and right direction. Save features in a table and save table in database. 


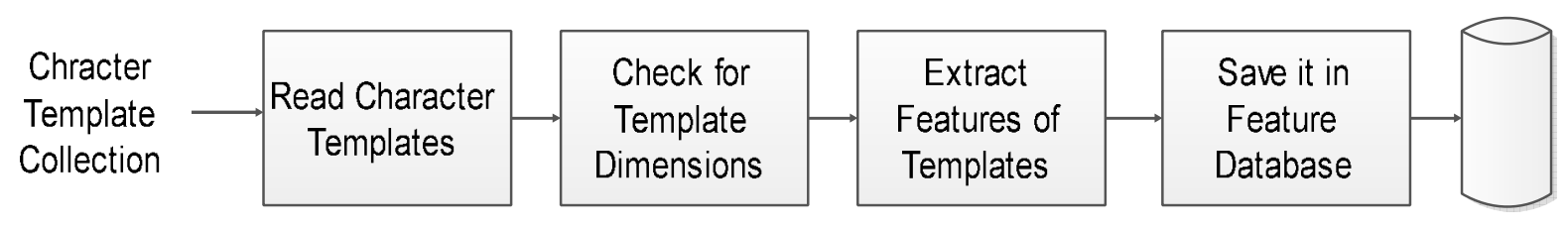

Fig. 2.1 Block Diagram of Training Process

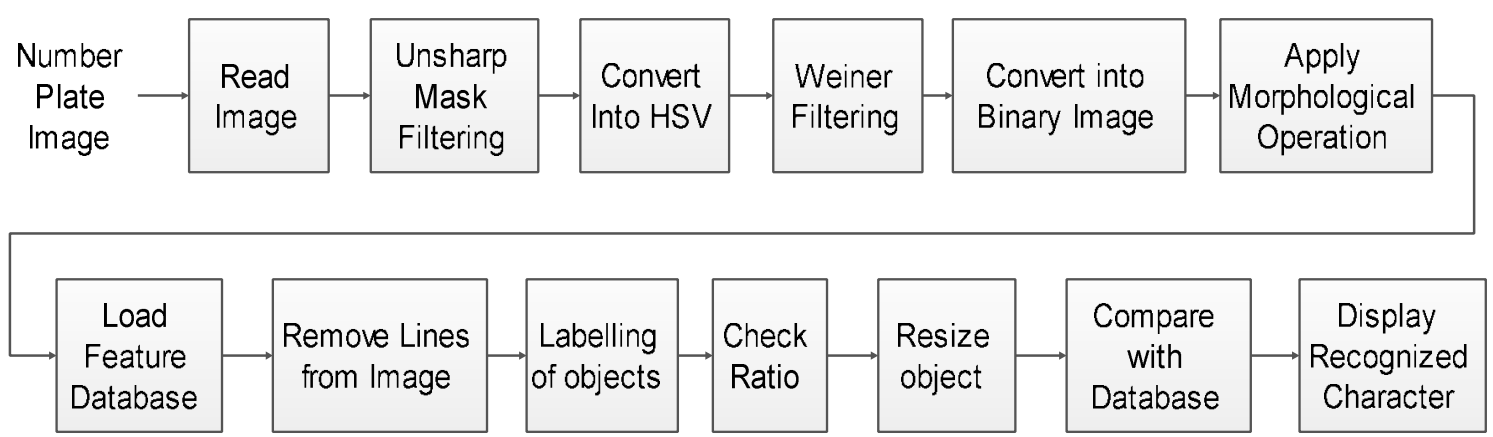

Fig. 2.2 Block Diagram of Character Recognition Process

The block diagram of the recognition process is shown in the Fig. 2.2. The main block diagram is the number plate image given as input. The image can be taken by any digital camera approximately of 5MP.

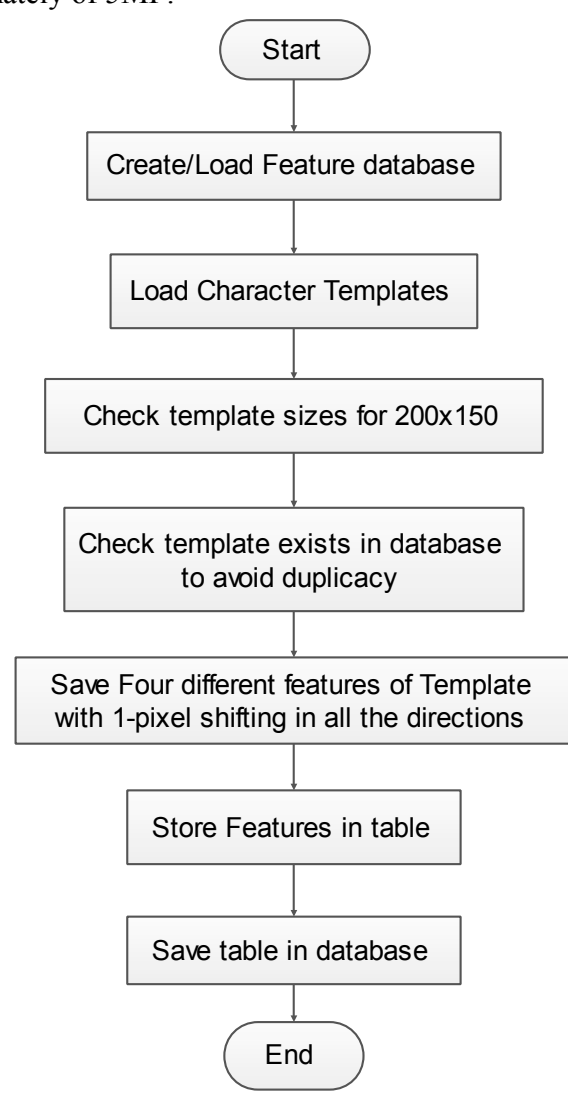

Fig. 2.3 Flow chart of Feature Database Preparation

\section{(Training) Process}

In the character recognition process the images are preprocessed by applying un sharp mask filtering followed by HSV colour space conversion followed by wiener filtering. After the image is converted into binary image, the image is passed through morphological operation.

After the morphological operation is applied, the feature database is loaded and labelling is performed in the binary image the labeled objects are checked for specified character ratio subsequently the features of the objects are compared with template features and the most similar character are printed. In the Fig. 2.3 shows the steps of the algorithm in simulation tool. The steps are as follows:

a)

b) a) Start of simulation

c) b) Create/Load Feature Database

d) c) Load Character Templates

e) d) Check template is in specific size or not.

f) e) Check whether template features already exists in the database or not

g) f) Save four different features including original template features

h) g) Store features in the Feature Database

i) h) Save Feature Database

j) The next step is the recognition process which is shown In the Fig. 2.4. The steps of the algorithm are as follows:
a) Start of simulation
b) Browse Number Plate Image 


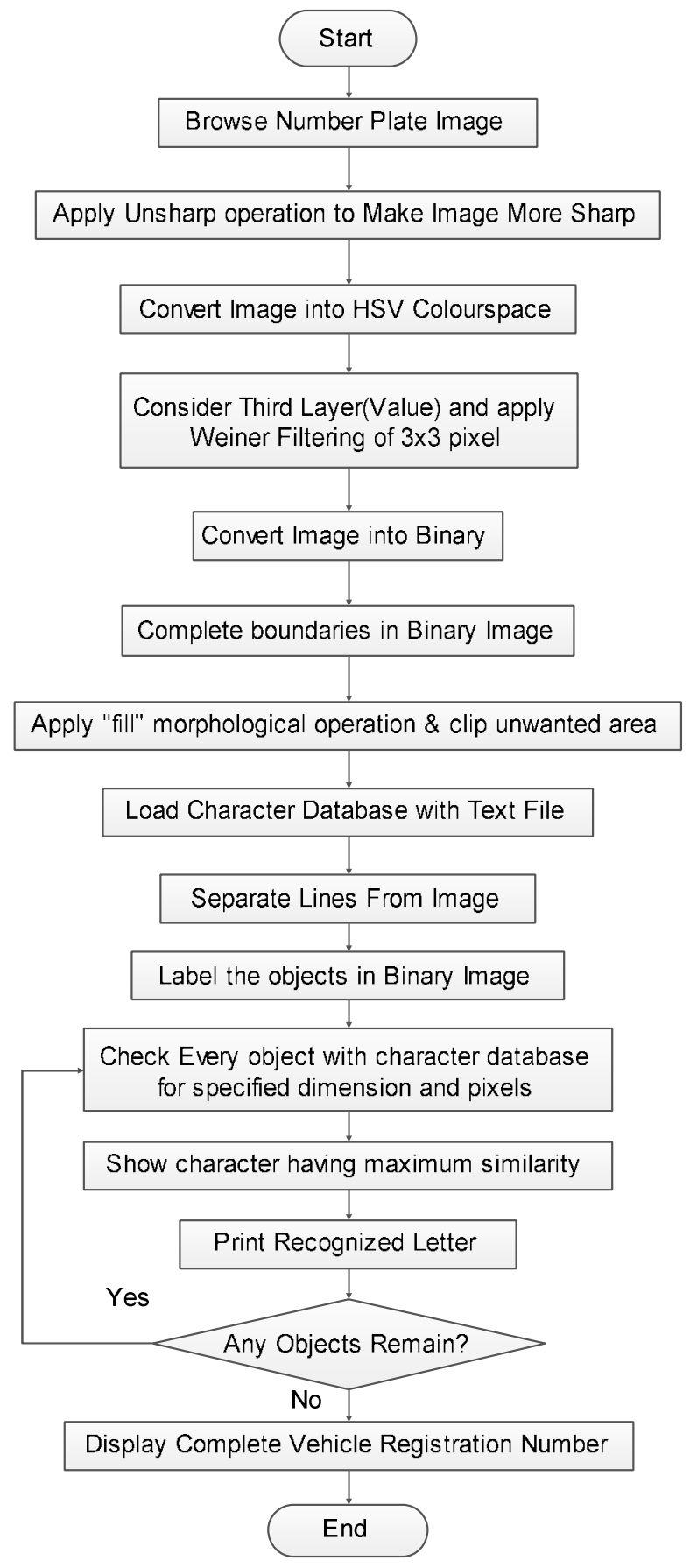

Fig. 2.4 Flow chart of Recognition Process

c) Applying Unsharp Masking to enhance the sharpness of the image

d) After that the image is converted in HSV colour space

e) Considering third layer and apply Weiner Filtering to remove Noises

f) Convert image into Binary to make character other objects in the image into black and white colours only

g) g) Applying boundaries completion operation to complete the object boundaries

h) Now "fill" using morphological operation to reduce the holes in the objects and clip unwanted area i) Load Character Feature Database and Text File to print recognize characters

j) Separate lines from image

k) Label the objects found in the image(group of pixels independent of each other)

1) Check every object with character database with specified dimension and pixels

m) Record character with maximum similarity

n) Print Recognized Character

o) If objects remained in the image go to step (1)

p) Display complete vehicle registration number

q) End of Simulation

\section{3. Simulation Results}

The character recognition process especially for Indian High Security Vehicle Number Plate recognition is not found out yet with optimum accuracy. The proposed algorithm in this paper is an example of the high quality algorithm to achieve better results with considerable speed.

The proposed approach can be implemented in various fields related to transport department or in any organization which deals with the vehicles. This algorithm does not need much investment to start the working system.

A camera and a personal computer is sufficient to start system work.

The simulation steps, outputs and results of algorithm at various stages are shown in the figures below. The outputs are as mentioned in the recognition steps in the previous section.

Fig. 3.1 is the original number plate image taken for recognition purpose. Fig. 3.2 is output after applying unsharp masking operation.

Fig. 3.3 is Value Layer after conversion in HSV colour space. Fig. 3.4 is denoised image after applying Weiner Filtering. Fig. 3.5 is a binary image of previous step.

Fig. 3.6 is the output of after boundary completion operation. Fig. 3.7 is the XOR operation of the boundary completed image and Binary Image.

Fig. 3.8 is output after applying morphological operation. Fig. 3.9 is the image after clipping of unwanted area.

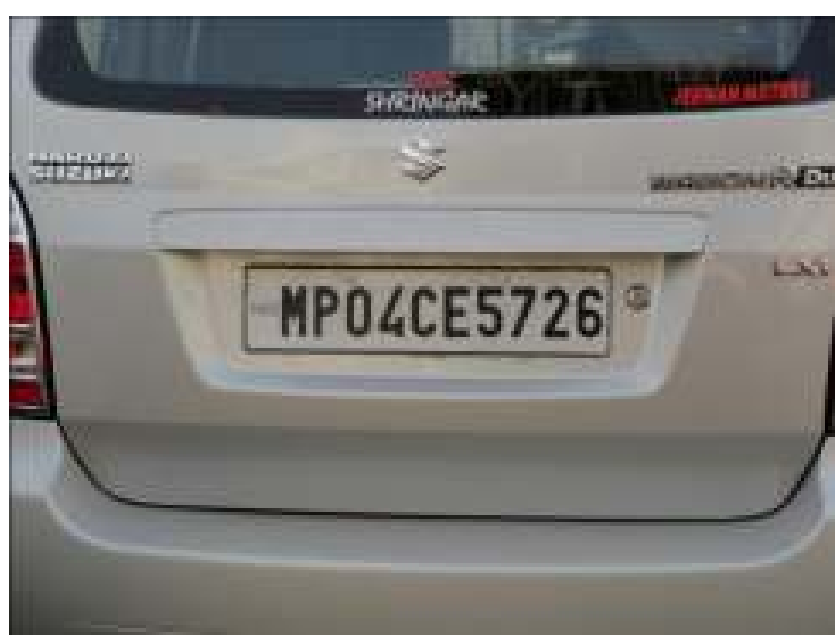

Fig. 3.1 Original High Security Number Plate 


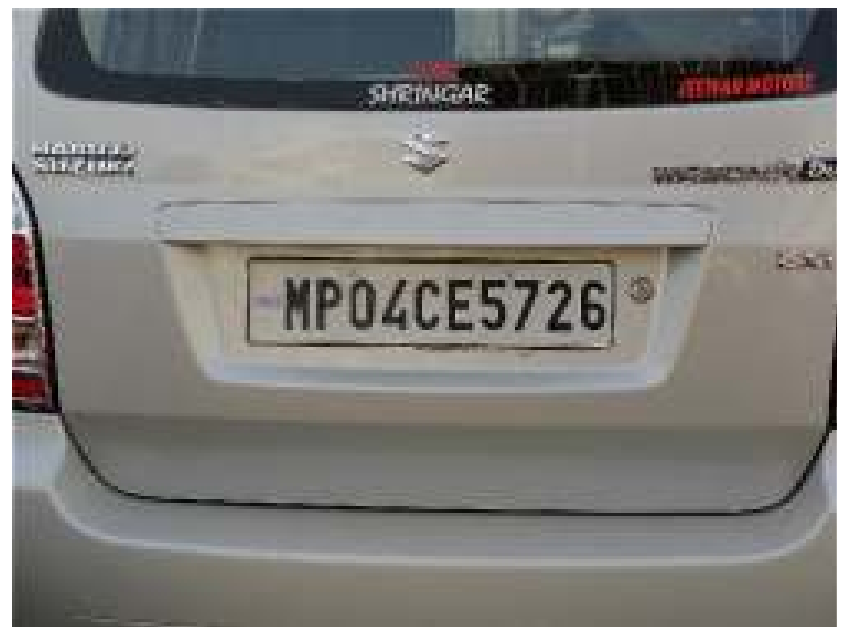

Fig. 3.2 Image after Unsharp Masking

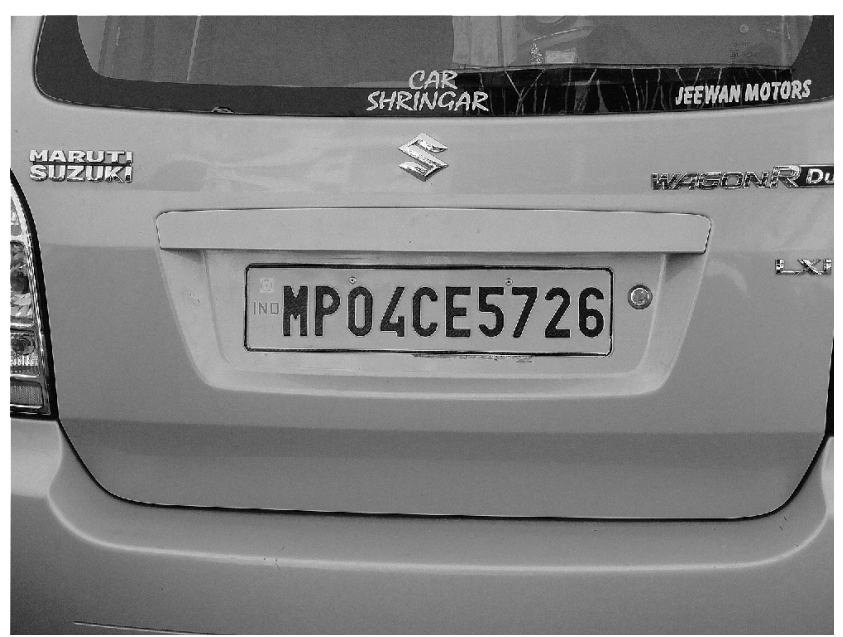

Fig. 3.3 HSV Image (Value Layer Only)

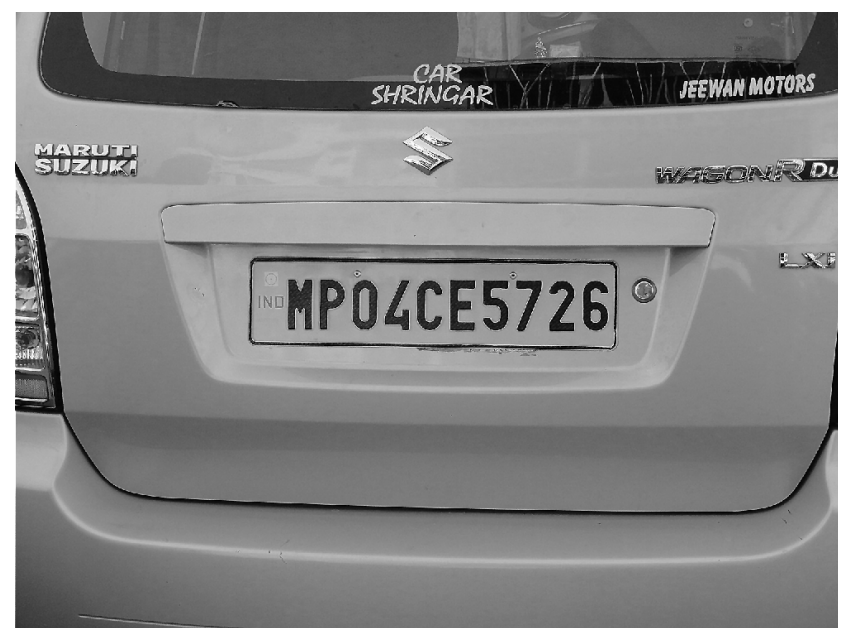

Fig. 3.4 Weiner Filtered Image

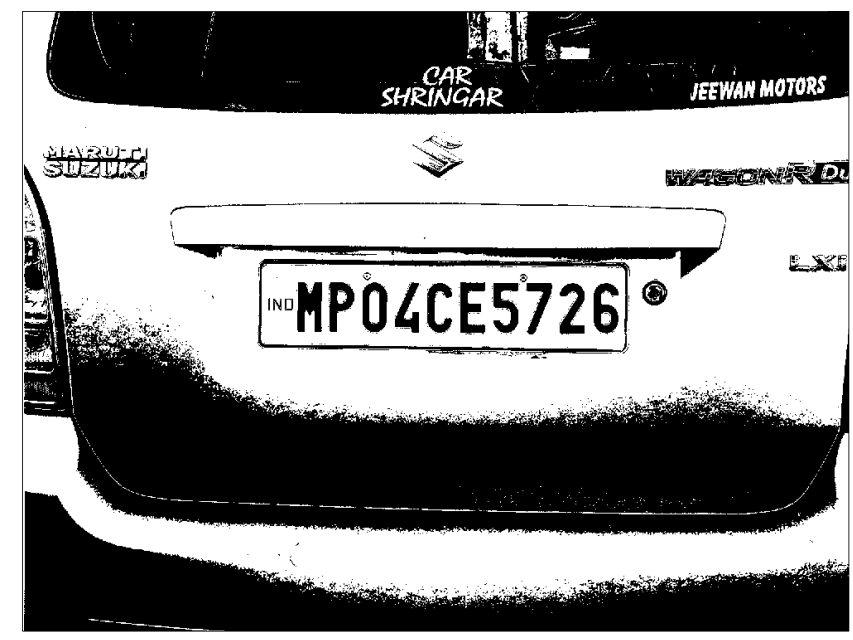

Fig. 3.5 Binary Image

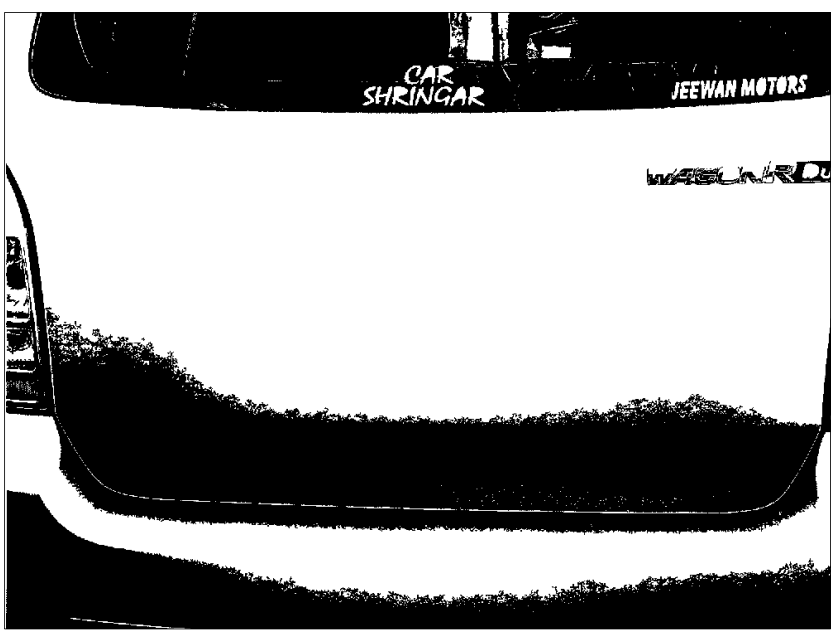

Fig. 3.6 Image after boundary completion

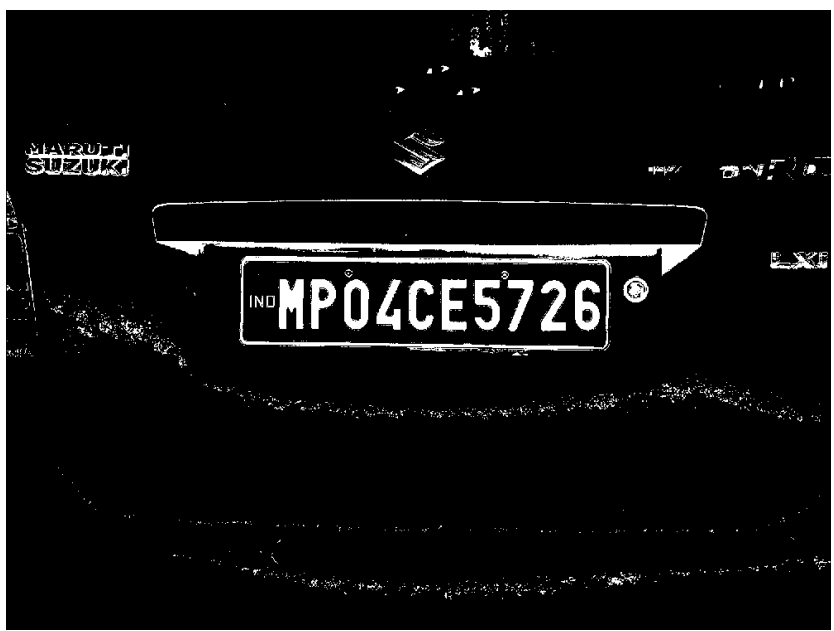

Fig. 3.7 XOR operation of boundary image and Binary Image 


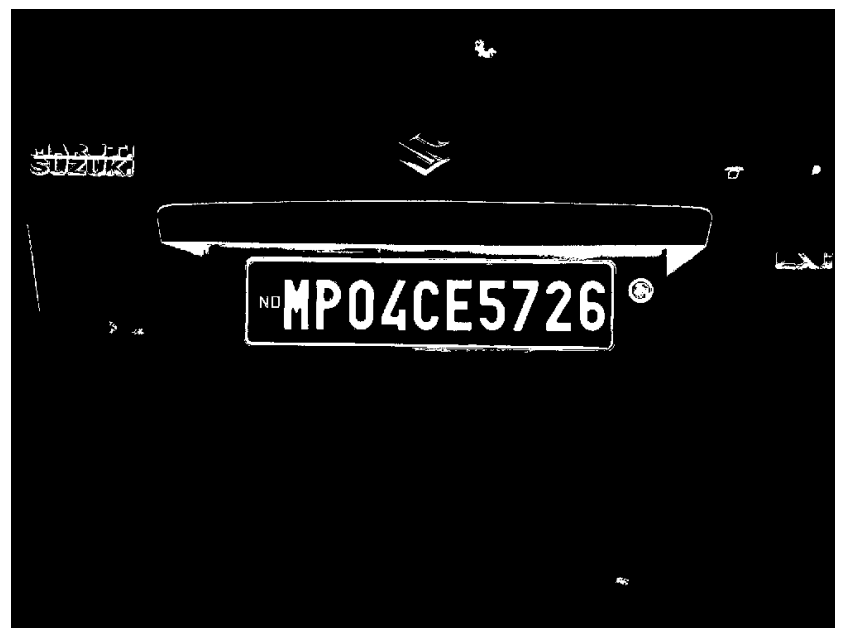

Fig. 3.8 Image after Morphological Operation

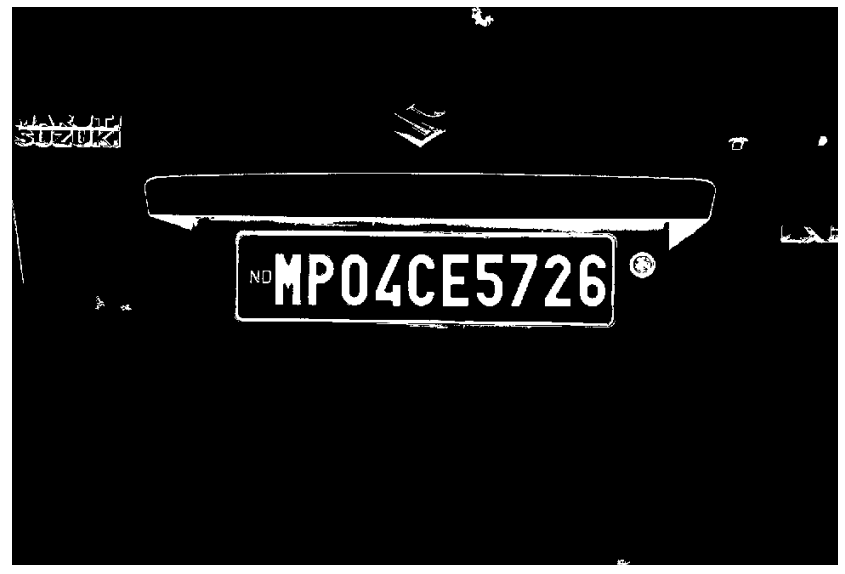

Fig. 3.9 Image after clipping unwanted area

Fig. 3.10 shows the image separated into lines. Fig. 3.11 shows the objects segmented as character. Fig. 3.12 shows the final recognized segmented characters. The recognized characters are recorded in a variable during simulation and printed on a text file. The screen shot of the text image is shown in the given Fig. 3.13 .

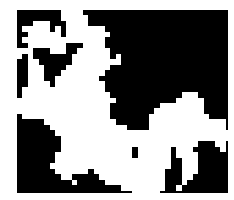

(a)

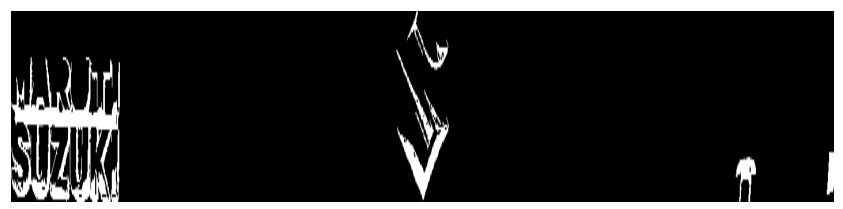

(b)

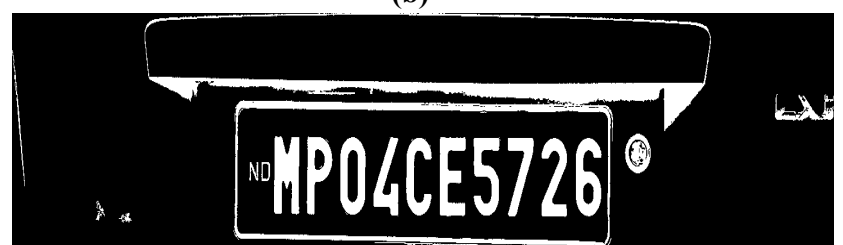

(c)

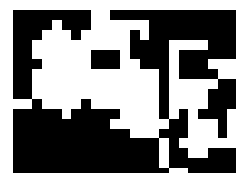

(d)

Fig. 3.10 After Lines separation operation (a), (b), (c) and (d)

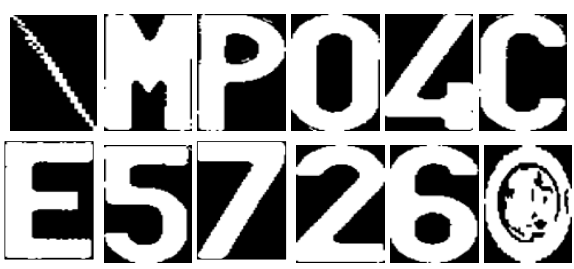

Fig. 3.11 Segmented and Extracted Objects (Algorithm Predicted like character)

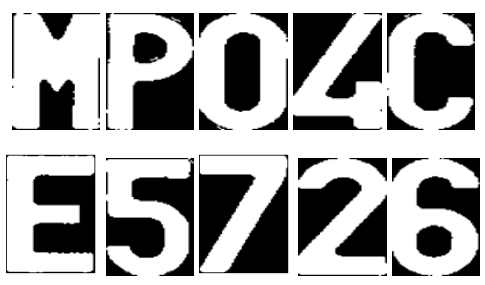

Fig. 3.12 Recognized Characters

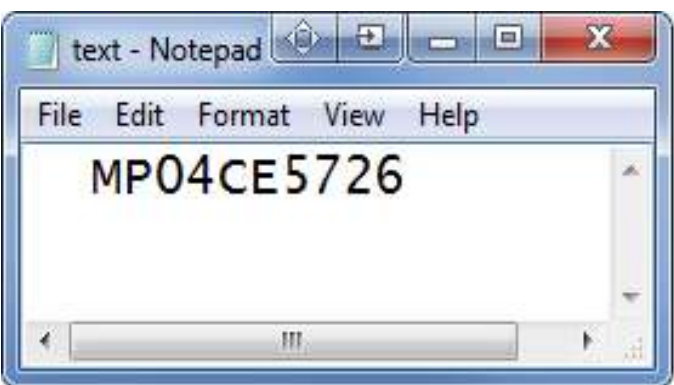

Fig. 3.13 Recognized Vehicle Registration Number

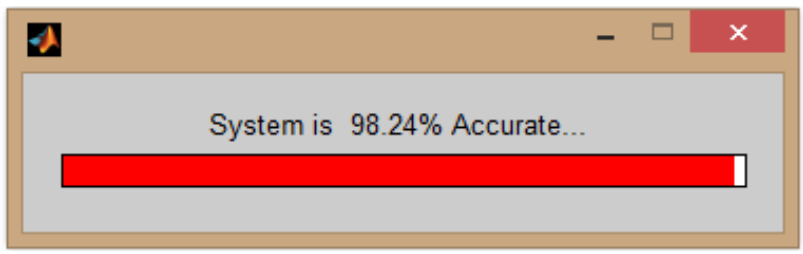

Fig. 3.14 Window showing Accuracy

\section{VALIDATION}

The validation part shows that our result is better than the base paper result. The below table shows the comparative analysis.

Table: 4.1 Comparative analysis between base paper \& published Paper

\begin{tabular}{|c|c|c|}
\hline PARAMETER & $\begin{array}{c}\text { BASE PAPER } \\
\text { RESULT } \\
{[1]}\end{array}$ & $\begin{array}{c}\text { OUR } \\
\text { RESULT }\end{array}$ \\
\hline$\%$ Efficiency & $\mathbf{1 5 0}$ & 170 \\
\hline
\end{tabular}




\section{CONCLUSION AND FUTURE SCOPE}

The outcomes of the steps of proposed algorithm for Indian High Security Number Plate Recognition are shown in the previous section. Fig 3.14 shows the accuracy of the algorithm which is about $97.08 \%$ more than the base paper which was $91.59 \%$. This accuracy is an achievement for the recognition systems. This vehicle number plate recognition experiment is performed on the images captured of vehicles around the Bhopal City. The algorithm is made adaptive in nature to make results accurate in case of the images taken from the long distances or from varied short distances. The results can be improved by adding more templates in the template database which can be obtained from the error templates.

Furthermore advancement can be made in terms of speed to detect and recognize the number plate in moving vehicles.

\section{REFERENCES}

[1] Ankush Roy, Debarshi Patanjali Ghoshal, "Number Plate Recognition for Use in Different Countries Using an Improved Segmentation", Emerging Trends and Applications in Computer Science (NCETACS), March2011 2nd National Conference of IEEE.

[2] Dening Jiang; Mekonnen, T.M.; Merkebu, T.E.; Gebrehiwot, A., "Car Plate Recognition System," Intelligent Networks and Intelligent Systems (ICINIS), 2012 Fifth International Conference on, vol., no., pp.9,12, 1-3 Nov. 2012.
[3] Fajas, F.; Yousuf, F.; Remya, P.R.; Pavanan, A.P.; Ambadiyil, S.; Swaminathan, V., "Automatic Number Plate Recognition for indian standard number plates," Ultra Modern Telecommunications and Control Systems and Workshops (ICUMT), 2012 4th International Congress on, vol., no., pp.1026,1028, 3-5 Oct. 2012

[4] Bakar, N.A.; Nawawi, M.R.M.; Abdullah, A.R.; Noordin, A.; Musa, Z.; Xian, O.C., "Malaysian vehicle license plate recognition using double edge detection," Control System, Computing and Engineering (ICCSCE), 2012 IEEE International Conference on, vol., no., pp.422,426, 23-25 Nov. 2012.

[5] Qiu Chengqun, "Design of Automobile License Plate Recognition System Based on MATLAB and Fuzzy PID," Measuring Technology and Mechatronics Automation (ICMTMA), 2013 Fifth International Conference on, vol., no., pp.438,441, 16-17 Jan. 2013.

[6] Shastry, S.; Gunasheela, G.; Dutt, T.; Vinay, D.S.; Rupanagudi, S.R., "“i" — A novel algorithm for optical character recognition (OCR)," Automation, Computing, Communication, Control and Compressed Sensing (iMac4s), 2013 International Multi-Conference on, vol., no., pp.389,393, 22-23 March 2013.

[7] Rashid, A.E., "A fast algorithm for license plate detection," Signal Processing Image Processing \& Pattern recognition (ICSIPR), 2013 International Conference on, vol., no., pp.44,48, 7-8 Feb. 2013. 